\title{
In vitro assessment of the effectiveness of whitening dentifrices for the removal of extrinsic tooth stains
}

\section{Débora Alves Nunes Leite Lima ${ }^{(a)}$ André Luís Faria e Silva ${ }^{(a)}$ Flávio Henrique Baggio Aguiar(b) Priscila Christiane Suzy Liporoni(c) Egberto Munin ${ }^{(\mathrm{d})}$ Gláucia Maria Bovi Ambrosano(e) José Roberto Lovadino(f)}

(a) PhD Candidates; (b) Assistant Professor; (f)Chairperson - Department of Restorative Dentistry, Piracicaba Dental School, State University of Campinas, SP, Brazil.

(c) Assistant Professor, Department of Restorative Dentistry, Vale do Paraíba University, SP, Brazil.

(d) Chairperson, Biomedicine Engineering Research Center, Vale do Paraíba University, SP, Brazil.

(e) Assistant Professor, Department of Social Dentistry/Statistics, Piracicaba Dental School, State University of Campinas, SP, Brazil.

\begin{abstract}
This in vitro study evaluated the effectiveness of whitening dentifrices for the removal of extrinsic tooth stains. Twenty dental blocks (4 x $4 \mathrm{~mm}$ ), including enamel and dentine, removed from freshly extracted bovine incisors, were randomly divided into 4 groups: G1 - distilled water, G2 - Colgate, G3 - Crest Extra Whitening and G4 - Rapid White. In all specimens, the dentin was covered with colorless nail polish, and the enamel was left exposed. Next, the specimens were immersed in a solution of black tea, which was changed every $24 \mathrm{~h}$, for a period of 6 days. After this period, a photo-reflectance reading was taken (Time 1) with a spectrometer. The stained specimens were then submitted to linear brushing movements (5,000 cycles) using brushes (Oral B-Soft) coupled to an automatic toothbrushing machine, under a static axial load of $200 \mathrm{~g}$ and with a speed of 4 movements $/$ second, at $37^{\circ} \mathrm{C}$, with the dentifrice or water being injected every $60 \mathrm{~s}$. When toothbrushing ended, a second photo-reflectance reading was taken (Time 2). The results were submitted to two-criteria analysis of variance (ANOVA) and to the Tukey test $(\alpha=0.05)$. When the two times for a same group were compared, Time 2 presented the highest reflectance values with statistical difference only for G3 and G4. Among the dentifrices tested, only the Rapid White group differed from the control group, presenting the highest reflectance values. Only the whitening dentifrice Rapid White was effective for the removal of extrinsic stains.
\end{abstract}

Descriptors: Dentifrices; Spectrophotometry; Pigmentation;

Toothbrushing.

\section{Corresponding author:}

Débora Alves Nunes Leite Lima

Departamento de Odontologia Restauradora

Faculdade de Odontologia de Piracicaba

Universidade Estadual de Campinas

(Unicamp)

Av. Limeira, 901

Piracicaba - SP - Brazil

CEP: 13414-903

E-mail: debora1201@yahoo.com.br

Received for publication on Jun 23, 2006

Accepted for publication on Mar 20, 2007 


\section{Introduction}

Patients increasingly seek to have an attractive smile, as it is considered to be synonymous with health. This growing demand for enhanced esthetic appearance has led to great development of bleaching products. ${ }^{1}$ However, before any of the bleaching products available on the market can be used, it is first necessary to look into the etiology of dental discoloration. ${ }^{2}$

Tooth color is influenced by a combination of intrinsic and extrinsic staining. Extrinsic pigmentations can originate from a smoking habit, from the use of certain cationic agents, such as chlorexidine, and from a diet rich in coloring foods. ${ }^{3}$ When beverages, such as coffee and tea, are frequently consumed, colors are deposited on the plaque and biological film present on the enamel surface, leading to tooth discoloration. ${ }^{4}$

These extrinsic stains can be removed by professional prophylaxis. However, the patient's use of a dentifrice can also contribute to dental stain removal and prevent recurrent discoloration. ${ }^{5}$ The dentifrice's stain-removal property is firstly related to the abrasives present in its composition. ${ }^{6}$ It is important to point out that if, on one hand, an increased dentifrice abrasiveness leads to improved stain removal efficacy, on the other hand it increases tooth wear. ${ }^{7,8}$ Thus, dentifrices containing active ingredients specifically designed for tooth stain removal, known as whitening dentifrices, have been introduced on the market. ${ }^{9,10}$

These dentifrices usually have enzymes in their composition that help to break down the organic components of biological film and remove stains and bacterial plaque. ${ }^{11,12}$ Considering the presence of chromogenic bacteria, bacterial plaque removal also helps whitening teeth. ${ }^{5}$ Another common dentifrice component is detergent, especially sodium lauryl sulfate. It acts by lowering the surface tension of stainproducing molecules, thus disrupting their potential to bind to enamel. ${ }^{9}$ Some whitening dentifrices also contain low peroxide concentrations, with the aim of releasing free oxygen radicals. ${ }^{13}$ These radicals are responsible for an oxidation reaction, which is the principle of the bleaching technique used for extrinsic and intrinsic stain removal. ${ }^{9}$

Considering the fast development of new whiten- ing products and their growing use by patients, this study aimed to evaluate the effectiveness in vitro of whitening dentifrices for extrinsic tooth stain removal. The null hypothesis tested was that there is no difference between the dentifrices used in this study.

\section{Material and Methods}

Twenty bovine teeth, stored in a $0.1 \%$ thymol solution, were selected for this study in accordance with the color similarity that most of them presented. Very dark or light teeth that stood out against the others were excluded by the visual method. The teeth had their roots removed and, next, the crowns were cleaned with water slurry of pumice flour in a rubber prophylaxis cup at low speed and examined under a light microscope $(\mathrm{x} 4)$ to exclude those with cracks.

Every tooth crown was set in an acrylic square base, which was fixed to a precision low-speed water-cooled diamond saw (Imptech PC10, Equilam Lab. Equip., Diadema, SP, Brazil) with two parallel disks, spaced $4 \mathrm{~mm}$ from each other and perpendicular to the buccal surface of the tooth. Each tooth was cut in the incisogingival and in the mesiodistal directions, resulting in a $4 \times 4 \mathrm{~mm}$ block. The height of each block was also standardized at $3 \mathrm{~mm}$, measured with a digital caliper (727-6/150, Starret, Itu, SP, Brazil). Superficial enamel was wet polished with silicon carbide $(\mathrm{SiC})$ paper \#600 and \#1,200 grit until the surface was flattened, while trying to maintain the largest possible thickness of enamel.

Afterwards, these dental blocks were randomly distributed into four experimental groups, according to the dentifrice to be used in this experiment. In one of these experimental groups, the blocks were brushed without dentifrice, with distilled water only, this being considered the control group. The experimental groups were as follows: G1 - control (distilled water), G2 - Colgate Total Regular (Colgate-Palmolive Indústria e Comércio Ltda., S. B. Campo, SP, Brazil), G3 - Crest Extra Whitening (Procter \& Gamble, Cincinnati, OH, USA) and G4 - Rapid White (Rapid White Products, Tonawanda, NY, USA). The composition of each dentifrice is listed in Table 1.

The enamel blocks were extrinsically stained with a black tea solution. In all the specimens, the dentin was covered with colorless nail polish, and the 
enamel was left exposed. Next, the specimens were immersed in a standard solution of black tea, which was renewed every $24 \mathrm{~h}$, for 6 days (Figure 1). The tea solution was produced by boiling $1.6 \mathrm{~g}$ of black tea (black tea leaves, Leão Junior S.A., Curitiba, PR, Brazil) in $100 \mathrm{ml}$ of distilled water, for $5 \mathrm{~min}$ and filtering it through gauze to remove the tea leaves from the infusion. After this period, a photo-reflectance reading was taken (Time 1) with a spectrometer.

For the reflectance analysis, a Teflon sphere in the reflectance configuration was used. Reflectance is the luminous radiation portion that is reflected by the material under study. Before the bleaching procedure, the samples were placed in a sample carrier that comprises a spectrometer sphere to obtain the initial reading (baseline). The integrating sphere optical signal was captured by an optical fiber with $600 \mu \mathrm{m}$ in diameter attached to a white light source. The optical potency available in the optical fiber tip was $5 \mathrm{~mW}$, and this fiber was placed $2 \mathrm{~mm}$ from the reference pattern (Teflon diffuser) and this distance was kept to the sample (Figure 2).

The reflectance signal was confined inside the integrating sphere and from this a proportional signal fraction was collected for analysis in the spectrometer, where it underwent spectral dispersion through a diffraction grating. The dispersed signal was reflected to a CCD (Charge Coupled Device) camera that converted the optical signal into a digital signal, which was interpreted by the computer and exhibited as intensity $\mathrm{X}$ wavelength signal. The reflectance analysis data reading was made with the aid of a microcomputer, which makes the spectral measurement available to the user in a file, as a result of the wavelength. The measurements of each sample were dealt with in order to obtain the area given by the graph (\%).

After the initial photo-reflectance reading, the stained specimens were submitted to linear brushing. For that, the blocks were allocated to the toothbrushing machine (Equilabor, Piracicaba, SP, Brazil) and were fixed on the machine support with hot glue so that the vestibular surface of the sample was parallel to this support. The dentifrices used in the experiment were diluted in distilled water at a ratio of $1: 3$ by weight. This ratio was used in order to allow the solution to be injected into the toothbrushing machine without obstructing the syringe tip.

The stained specimens were submitted to linear

Table 1 - Dentifrices evaluated in the study.

\begin{tabular}{l|l|l|l}
\hline \multicolumn{1}{c|}{ Dentifrice } & \multicolumn{1}{|c|}{ Manufacturer } & $\begin{array}{c}\text { Batch } \\
\text { Number }\end{array}$ & \multicolumn{1}{c}{$\begin{array}{c}\text { Composition } \\
\text { (as disclosed by the manufacturer) }\end{array}$} \\
\hline $\begin{array}{l}\text { Crest Extra } \\
\text { Whitening }\end{array}$ & Procter \& Gamble & 3294 GE & $\begin{array}{l}\text { Hydrated silica, water, sorbitol, glycerin, Polyoxyethylene (6), tetrasodium } \\
\text { pyrophosphate, sodium bicarbonate, sodium lauryl sulfate, poloxamer 407, flavor, } \\
\text { cellulose gum, sodium carbonate, sodium saccharin, carnauba wax, titanium dioxide. }\end{array}$ \\
\hline Rapid White & Rapid White Products & 235981 & $\begin{array}{l}\text { Water, hydrogen peroxide, hydrated silica, glycerin, sorbitol, pentasodium } \\
\text { triphosphate, Polyvinylpyrrolidone, titanium dioxide, cellulose gum, sodium lauryl } \\
\text { sulfate, sodium methyl cocoyl taurate, sodium benzoate, aroma, sodium saccharin, } \\
\text { sodium fluoride, sodium hydroxide. }\end{array}$ \\
\hline $\begin{array}{l}\text { Colgate } \\
\text { Total Regular }\end{array}$ & $\begin{array}{l}\text { Colgate-Palmolive } \\
\text { Indústria e Comércio Ltda. }\end{array}$ & BR122U & $\begin{array}{l}\text { Water, sorbitol, hydrated silica, sodium lauryl sulfate, Methyl vinyl ether/maleic } \\
\text { anhydrid and copolymer, aroma, carrageenan, sodium hydroxide, sodium fluoride, } \\
\text { triclosan, sodium saccharin. }\end{array}$ \\
\hline
\end{tabular}

Figure 1 - The enamel of the specimens was stained with a black tea solution.

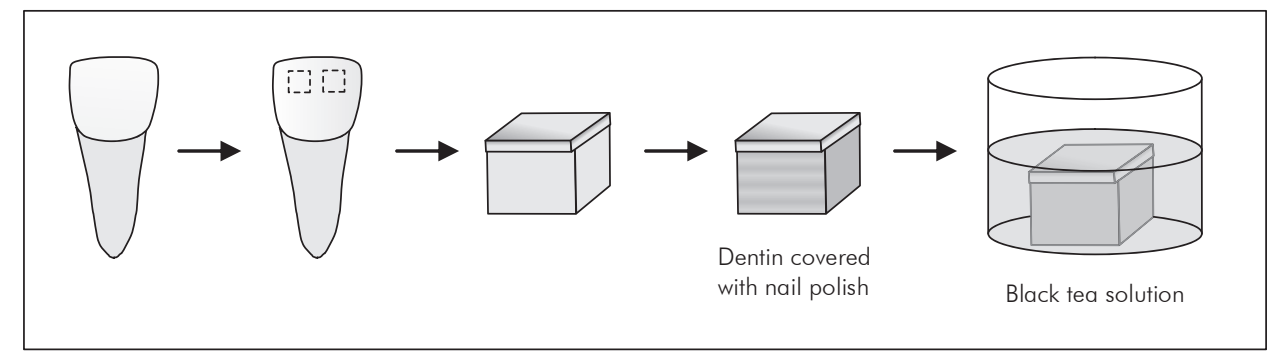



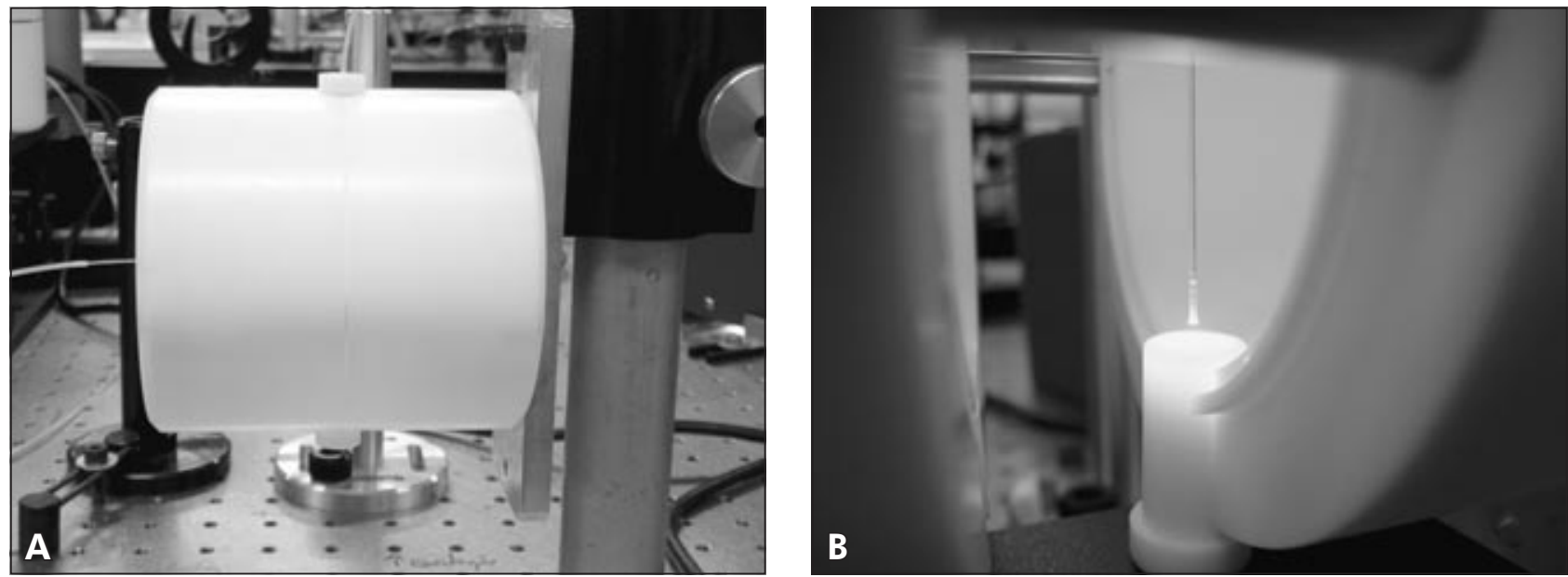

Figure 2 A and B - Positioning of the sample port and optical fiber inside the Teflon sphere in the reflectance analysis set.

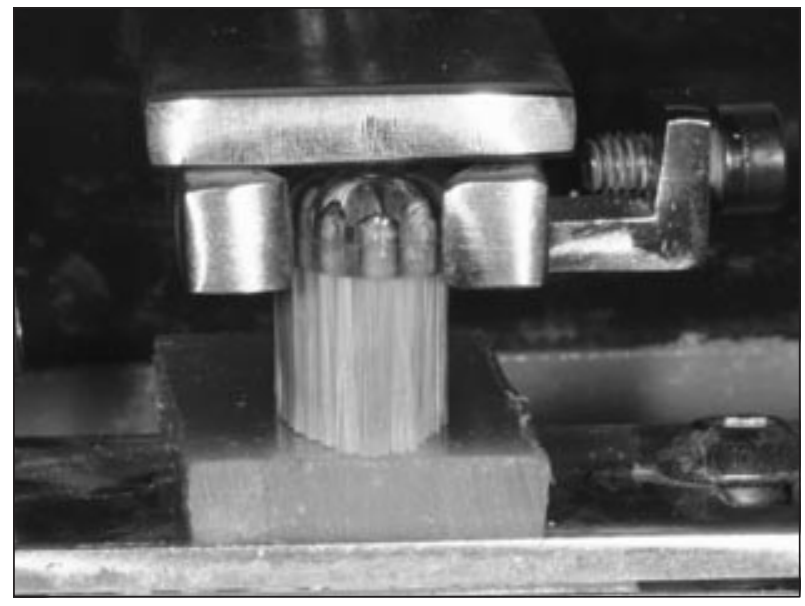

Figure 3 - Automatic toothbrushing machine used in the experiment.

brushing movements using toothbrush heads (Oral-B Indicator 40 Soft, Gillette do Brasil Ltda., Manaus, AM, Brazil) coupled to an automatic toothbrushing machine (Figure 3), under static axial load of $200 \mathrm{~g}$ and with a speed of 5 movements/second, at $37^{\circ} \mathrm{C}$, the dentifrice or water being injected every $60 \mathrm{~s}$. Five thousand brushing cycles were accomplished, which is equivalent to approximately 6 months of toothbrushing.

When toothbrushing ended, the specimens were removed from the toothbrushing machine and were washed with water spray until the dentifrice residues had been removed. A new photo-reflectance reading was taken (Time 2).
The results were submitted to two-criteria analysis of variance (ANOVA), the factors being dentifrice and time, and to the Tukey test at a 5\% significance level.

\section{Results}

The analysis of variance showed no significant effect for the dentifrice factor alone $(\mathrm{p}=0.28)$, but for the time factor $(\mathrm{p}<0.01)$ and for the dentifrice and time interaction $(\mathrm{p}=0.02)$ the differences were statistically significant. The results for the Tukey test are shown in Table 2.

Before brushing, there was no significant difference for the reflectance values among any of the studied groups. After brushing, Rapid White presented higher reflectance values, although differing significantly only from the distilled water group. At this Time, the other two dentifrices used, Regular Colgate and Crest Whitening, did not differ from any of the other groups.

For the same group, there were statistically significant differences between the values of the reading taken after brushing and those of the first reading only for Rapid White and Crest Whitening. For distilled water and Regular Colgate, there was no significant difference between the two times of reading.

\section{Discussion}

Tooth color is the result of the behavior of incident light on its surface. Part of the incident light on 
Table 2 - Reflectance Reading Results.

\begin{tabular}{l|c|c}
\hline \multirow{2}{*}{\multicolumn{1}{c|}{ Group }} & \multicolumn{2}{c}{ Time } \\
\cline { 2 - 3 } & Before brushing & After brushing \\
\hline Distilled water & $44.47(18.65) \mathrm{A} \mathrm{a}$ & $54.86(20.22) \mathrm{A} \mathrm{b}$ \\
\hline Colgate Regular & $51.94(17.87) \mathrm{A} \mathrm{a}$ & $70.52(33.54) \mathrm{A} \mathrm{ab}$ \\
\hline Crest Whitening & $43.58(14.27) \mathrm{A} \mathrm{a}$ & $88.89(33.54) \mathrm{B} \mathrm{ab}$ \\
\hline Rapid White & 46.97 (19.29) A a & 112.27 (43.65) B a \\
\hline
\end{tabular}

Reflectance mean (\%) and standard deviation. Mean values followed by different letters differ among them for the Tukey test $(p<0.05)$ (capital letters in lines and lower case letters in rows).

the tooth scatters, while the other part is absorbed by pigmented proteins and other pigments present in the tooth. The greater the increase in the amount of these pigments, the greater is the absorption of the incident light and the darker the tooth becomes. ${ }^{14,15}$

Many methods are currently used to assess tooth color, the objective methods such as reflectance spectrophotometer analysis being the most reliable. ${ }^{16,17}$ Spectrophotometers and colorimeters are instruments commonly used to measure the color of an object. Spectrophotometers differ from colorimeters in that they measure the reflectance of light within the entire visible spectrum, whereas colorimeters measure reflected light in only three wavelengths: red, green and blue. ${ }^{15}$ In the present study, the color alteration measurements of the specimens were taken by a reflectance spectrophotometer. For this, a light was made to fall on the enamel surface of each specimen through an optical fiber, the entire reflected portion of the light being caught by the device and then quantified. Thus, as the tooth got whiter, a lower amount of light was absorbed and the reflectance value was higher.

To standardize the initial color of the specimens, a solution of black tea was used. The method used in this study for simulated extrinsic tooth staining was demonstrated to be effective. At the end of tooth specimen immersion in the black tea solution, the reflectance reading disclosed a similarity in the color of dental enamel among the specimens, without statistical difference between groups. Sulieman et al. ${ }^{18}$ (2003) also used black tea for six days to create staining in dental specimens, which permitted tooth bleaching effectiveness to be assessed. In their study, the authors also developed an intrinsic dentin pigmentation staining. In the present study, however, contact with the tea solution was restricted to the enamel only.

According to Sexson, Phillips ${ }^{19}$ (1951), for each toothbrushing session a patient performs, approximately 15 cycles are executed in a determined area. Thus, in two daily toothbrushing sessions, 10,000 cycles are performed at the end of one year. In the present study, 5,000 brushing cycles were performed, which is equivalent to approximately 6 months of toothbrushing. The majority of studies that evaluate dentifrice whitening effects are conducted in a period that varies from 2 weeks to 6 months. ${ }^{12}$ Thus, a relatively long time of brushing was simulated in this study, seeking to optimize the whitening effect of the dentifrices used. It is also important that during brushing, dentifrice was injected every $60 \mathrm{~s}$, which allowed the active dentifrice components, among them the abrasives, to be renewed.

When the reflectance values for both of the tested times are compared, it was found that the whitening dentifrices, Crest Whitening and Rapid White, presented higher reflectance values than the conventional dentifrice (Colgate Regular) after the brushing protocol. These whitening dentifrices have enzymes and detergents in their compositions, which are thought to help with stain removal. Comparing all the dentifrices, the Rapid White was the only one to statistically differ from the control group (distilled water). Rapid White has a small amount of hydrogen peroxide, which releases free radical oxygen capable of combining with the stain molecule, thus removing it. However, this component stays in contact with the tooth for a short period of time, which limits its action.

The whitening effect can be attributed to the extrinsic stain removal and also to the change in the reflection of the light. ${ }^{14}$ According to Wulknitz ${ }^{13}$ (1997), hydrated silica has great cleaning ability and, consequently, stain removal ability. All the dentifrices tested present hydrated silica in their compositions. The incorporation of abrasives in dentifrices might help physically remove stain, thus some degree of stain removal may be expected even with regular 
products. ${ }^{20}$ The two whitening dentifrices tested in the experiment did not differ from the regular one as regards reflectance.

Crest Whitening also has sodium bicarbonate as abrasive. Kleber et al. ${ }^{21}$ (1998) investigated the whitening effect of toothbrushing with different concentrations of sodium bicarbonate-based dentifrices and concluded that the dentifrices that presented this type of abrasive were more efficient for stain removal. In the present study, Crest Whitening did not significantly differ from Rapid White and Colgate Regular as regards reflectance values.

It is important to note that in addition to the type of abrasive, the amount of this component has a direct relation to dentifrice abrasiveness. The abrasive

\section{References}

1. Gerlach RW, Barker ML. Clinical response of three direct-toconsumer whitening products: strips, paint-on gel, and dentifrice. Compend Contin Educ Dent. 2003;24(6):458, 461-4, 466 passim.

2. Hattab FN, Qudeimat MA, al-Rimawi HS. Dental discoloration: an overview. J Esthet Dent. 1999;11(6):291-310.

3. Matheson JR, Cox TF, Baylor N, Joiner A, Patil R, Karad V et al. Effect of toothpaste with natural calcium carbonate/perlite on extrinsic tooth stain. Int Dent J. 2004;54(5 Suppl 1):3215.

4. Watts A, Addy M. Tooth discolouration and staining: a review of the literature. Br Dent J. 2001;190(6):309-16.

5. Claydon NC, Moran J, Bosma ML, Shirodaria S, Addy M, Newcombe R. Clinical study to compare the effectiveness of a test whitening toothpaste with a commercial whitening toothpaste at inhibiting dental stain. J Clin Periodontol. 2004;31(12):1088-91.

6. Meyers IA, McQueen MJ, Harbrow D, Seymour GJ. The surface effect of dentifrices. Aust Dent J. 2000;45(2):118-24.

7. Koertge TE. Management of dental staining: can low-abrasive dentifrices play a role? Compend Contin Educ Dent. 1997;18(21 Suppl):33-8; quiz 47.

8. Pickles MJ. Tooth wear. Monogr Oral Sci. 2006;19:86-104.

9. Pontefract H, Sheen S, Moran J. The benefits of toothpaste real or imagined? Review of its role in tooth whitening. Dent Update. 2001;28(2):67-70, 72, 74.

10. Yankell SL, Emling RC, Petrone ME, Rustogi K, Volpe AR, DeVizio W et al. A six-week clinical efficacy study of four commercially available dentifrices for the removal of extrinsic tooth stain. J Clin Dent. 1999;10(3 Spec No):115-8.

11. Lynch E, Samarawickrama DY, Claxson AW, Hawkes JE, Atherton M, Naughton DP et al. Safety aspects concerning the should ideally provide stain removal without causing wear of the tooth. ${ }^{6}$ Therefore, the results of this study must be viewed with caution, since only the whitening effect of two whitening dentifrices was assessed. The enamel wear caused by these dentifrices still needs to be assessed before they are recommended for routine use.

\section{Conclusion}

Only the whitening dentifrice Rapid White was effective for the removal of extrinsic stains.

\section{Acknowledgements}

Oral-B (Gillette do Brasil Ltda.) kindly provided the toothbrushes used in this research.

therapeutic and cosmetic applications of hydrogen peroxide $\left(\mathrm{H}_{2} \mathrm{O}_{2}\right)$-containing gels, whiteners, oral rinses and dentifrices. J Ir Dent Assoc. 1994;40(3):78-82.

12. Walsh TF, Rawlinson A, Wildgoose D, Marlow I, Haywood $\mathrm{J}$, Ward JM et al. Clinical evaluation of the stain removing ability of a whitening dentifrice and stain controlling system. J Dent. 2005;33(5):413-8.

13. Wulknitz P. Cleaning power and abrasivity of European toothpastes. Adv Dent Res. 1997;11(4):576-9.

14. ten Bosch JJ, Coops JC. Tooth color and reflectance as related to light scattering and enamel hardness. J Dent Res. 1995;74(1):374-80.

15. Chu SJ. Use of a reflectance spectrophotometer in evaluating shade change resulting from tooth-whitening products. J Esthet Restor Dent. 2003;15(Suppl 1):42-8.

16. Horn DJ, Bulan-Brady J, Hicks L. Sphere spectrophotometer versus human evaluation of tooth shade. J Endod. 1998;24(12):786-90.

17. Joiner A. Tooth colour: a review of the literature. J Dent. 2004;32(Suppl 1):3-12.

18. Sulieman M, Addy M, Rees JS. Development and evaluation of a method in vitro to study the effectiveness of tooth bleaching. J Dent. 2003;31(6):415-22.

19. Sexson JC, Phillips RW. Studies on the effects of abrasives on acrylic resins. J Prosthet Dent. 1951;1(4):454-71.

20. Amaral CM, Rodrigues JA, Erhardt MC, Araujo MWB, Marchi GM, Heymann $\mathrm{H}$ et al. Effect of whitening dentifrices on the superficial roughness of esthetic restorative materials. J Esthet Restor Dent. 2006;18(2):111-8.

21. Kleber CJ, Moore MH, Nelson BJ. Laboratory assessment of tooth whitening by sodium bicarbonate dentifrices. J Clin Dent. 1998;9(3):72-5. 